\title{
The Approximate Rectangle of Influence Drawability Problem
}

\author{
Emilio Di Giacomo ${ }^{1}$, Giuseppe Liotta ${ }^{1}$, and Henk Meijer ${ }^{2}$ \\ ${ }^{1}$ Dip. di Ingegneria Elettronica e dell'Informazione, Università degli Studi di Perugia \\ \{digiacomo, liotta\}@diei.unipg.it \\ 2 Roosevelt Academy, The Netherlands \\ h.meijer@roac.nl
}

\begin{abstract}
We prove that all planar graphs have an open/closed $\left(\varepsilon_{1}, \varepsilon_{2}\right)$-rectangle of influence drawing for $\varepsilon_{1}>0$ and $\varepsilon_{2}>0$, while there are planar graphs which do not admit an open/closed $\left(\varepsilon_{1}, 0\right)$-rectangle of influence drawing and planar graphs which do not admit a $\left(0, \varepsilon_{2}\right)$-rectangle of influence drawing. We then show that all outerplanar graphs have an open/closed $\left(0, \varepsilon_{2}\right)$-rectangle of influence drawing for any $\varepsilon_{2} \geq 0$. We also prove that if $\varepsilon_{2}>2$ an open/closed $\left(0, \varepsilon_{2}\right)$-rectangle of influence drawing of an outerplanar graph can be computed in polynomial area. For values of $\varepsilon_{2}$ such that $\varepsilon_{2} \leq 2$, we describe a drawing algorithm that computes $\left(0, \varepsilon_{2}\right)$-rectangle of influence drawings of binary trees in area $O\left(n^{2+f\left(\varepsilon_{2}\right)}\right)$, where $f\left(\varepsilon_{2}\right)$ is a logarithmic function that tends to infinity as $\varepsilon_{2}$ tends to zero, and $n$ is the number of vertices of the input tree.
\end{abstract}

\section{Introduction}

A proximity drawing of a graph is a straight-line drawing that satisfies a set of proximity constraints. The proximity constraints apply to each pair $u, v$ of vertices in the drawing and establish whether $u, v$ are close to one another according to some definition of closeness. A typical approach to define the closeness of $u, v$ in a proximity drawing is to use the concept of region of influence, defined as a suitable region of the plane whose size only depends on the coordinates of $u$ and $v$. Vertices $u$ and $v$ are said to be close if and only if their region of influence is empty, i.e., it does not contain other vertices of the drawing except than, possibly, $u$ and $v$ themselves. In a strong proximity drawing two vertices are adjacent if and only if they are close to one another. A weak proximity drawing only guarantees the closeness of any pair of adjacent vertices, while the region of influence of non-adjacent vertices may or may not be empty (see, e.g. [5/7/12]).

Clearly, by varying the definition of region of influence and by adopting either the strong or the weak definition of proximity drawing, a same graph may or may not be representable. For example, the relative neighborhood region of $u, v$ is the intersection of the two disks having center at $u$ and at $v$ and with radius the distance $d(u, v)$. It is known that all trees having vertices of degree at most five admit a strong proximity drawing such that the region of influence is the relative neighborhood region. However, if the closeness is defined by using the disk having $u$ and $v$ as antipodal points (the well-known Gabriel region of $u, v$ ) then a tree with vertices of degree five does not have a strong proximity drawing [6]. On the other hand, all trees have a weak proximity

W. Didimo and M. Patrignani (Eds.): GD 2012, LNCS 7704, pp. 114-125, 2013.

(C) Springer-Verlag Berlin Heidelberg 2013 
drawing with both the Gabriel region and the relative neighborhood region [7]. See also [12] for a survey on the proximity drawability problem.

The notions of strong proximity drawing and of weak proximity drawing have been recently extended by Evans et al. who introduce the concept of approximate proximity drawing, also called $\left(\varepsilon_{1}, \varepsilon_{2}\right)$-proximity drawing [9]. Intuitively, given two real non negative numbers $\varepsilon_{1}$ and $\varepsilon_{2}$ and a definition of region of influence, an $\left(\varepsilon_{1}, \varepsilon_{2}\right)$-proximity drawing is such that for any pair of adjacent vertices their region of influence "shrunk" by the factor $\frac{1}{1+\varepsilon_{1}}$ is empty, while for any pair of non-adjacent vertices their region of influence "expanded" by the factor $\left(1+\varepsilon_{2}\right)$ is not empty. By making $\varepsilon_{1}$ and $\varepsilon_{2}$ arbitrarily small, it is possible to arbitrarily approximate a strong proximity drawing; namely, a strong proximity drawing is an $\left(\varepsilon_{1}, \varepsilon_{2}\right)$-proximity drawing such that $\varepsilon_{1}=\varepsilon_{2}=0$. Also, a $(0, \infty)$-proximity drawing is such that any two adjacent vertices have their region of influence empty, while for any pair of non-adjacent vertices their proximity region is extended to the whole plane and it is never empty (for graphs with at least three vertices). Hence, a weak proximity drawing is a $(0, \infty)$-proximity drawing.

In this paper we study approximate rectangle of influence drawings, i.e., approximate proximity drawings whose region of influence is the rectangle of influence. The rectangle of influence of two points $u$ and $v$ is the axis-aligned rectangle having $u$ and $v$ at diagonally opposite corners. Besides graph drawing applications, rectangles of influence have been studied in the context of rectangular visibility that has applications, for example, in art gallery and pattern recognition problems (see, e.g., [411]1]). Depending on whether the rectangle of influence of $u$ and $v$ is assumed to be an open or a closed set, the graph drawing literature distinguishes between (strong or weak) open rectangle of influence drawings and closed rectangle of influence drawings, also called open RIDs and closed RIDs, for short.

Compared with weak rectangle of influence drawings, $\left(\varepsilon_{1}, \varepsilon_{2}\right)$-RIDs not only guarantee that adjacent vertices are relatively close to one another, but also have the desirable aesthetic property that pairs of non-adjacent vertices are relatively far apart. Compared with strong RIDs, $\left(\varepsilon_{1}, \varepsilon_{2}\right)$-rectangle of influence drawings have the advantage of significantly enlarging the family of the representable graphs. In order to help better locating our results within the existing literature, we briefly recall some of the most relevant papers about strong and weak rectangle of influence drawings.

Classes of graphs that admit open or closed strong rectangle of influence drawings are characterized in [13]. The paper shows that even for structurally simple planar graphs such as cycles, wheels, trees, and outerplanar graphs, the existence of open/closed RIDs imposes severe restrictions on the combinatorial properties that the representable graphs must satisfy. On the other hand, Biedl et al. [5] show that all planar graphs without filled 3-cycles (i.e., planar embedded graphs such that the interior of every 3-cycle does not contain vertices) have a weak RID both with the open and with the closed rectangle of influence. They also show that a planar graph has a weak closed RID if and only if it is without filled 3-cycles, and leave unanswered the question of characterizing those planar graphs that have a weak open RID. Miura, Matsuno, and Nishizeki [14] partially answer this question by characterizing those triangulated plane graphs that admit an open weak rectangle of influence drawing. They also give a sufficient condition for the weak open rectangle of influence drawability of the inner triangulated plane 
graphs, expressed in terms of labeling of angles of a suitable subgraph, called frame graph. The frame graph is obtained by removing all vertices and edges in the proper inside of every maximal filled triangle. Alamdari and Biedl [1] further elaborate on the ideas by Miura, Matsuno, and Nishizeki and characterize the inner triangulated plane graphs that admit a weak open rectangle of influence drawing such that no two vertices of the frame graph have the same $x$-coordinate or the same $y$-coordinate. A significant research effort has also been devoted to the area required by open/closed weak rectangle of influence drawings, including [5 15 16 [18 19$]$.

The main goal of this paper is to establish what graphs admit an $\left(\varepsilon_{1}, \varepsilon_{2}\right)$-rectangle of influence drawing for different values of $\varepsilon_{1}$ and $\varepsilon_{2}$ and to design area efficient algorithms for some graph families. Our results can be listed as follows.

- We show in Section 2 that every planar graph has an open/closed $\left(\varepsilon_{1}, \varepsilon_{2}\right)$-RID for every $\varepsilon_{1}, \varepsilon_{2}>0$. Also, we show that if one of the two parameters $\varepsilon_{1}$ and $\varepsilon_{2}$ is equal to 0 , not all planar graphs admit an open/closed $\left(\varepsilon_{1}, \varepsilon_{2}\right)$-RID. These results extend to approximate rectangle of influence drawings similar results that Evans et al. [9] proved for other types of approximate proximity drawings.

- In Section 3 we study the $\left(\varepsilon_{1}, \varepsilon_{2}\right)$-rectangle of influence drawability for $\varepsilon_{1}=0$ and $\varepsilon_{2}>0$. We concentrate on outerplanar graphs and prove that they admit a $\left(0, \varepsilon_{2}\right)$-RID for any positive value of $\varepsilon_{2}$. Note that if the region of influence is the Gabriel region and $\varepsilon_{2}<2$, not all outerplanar graphs admit an $\left(0, \varepsilon_{2}\right)$-proximity drawing [9]. Furthermore, it is known that even simple outerplanar graphs do not have a $(0,0)$-RID, while every outerplanar graph has a $(0, \infty)$-RID [5[13].

- Since the drawing technique of the bullet above requires exponential area, we devote Section 4 to computing area efficient $\left(0, \varepsilon_{2}\right)$-RIDs of outerplanar graphs. We prove that if $\varepsilon_{2}>2$ an open/closed $\left(0, \varepsilon_{2}\right)$-rectangle of influence drawing of an outerplanar graph can be computed in polynomial area. For values of $\varepsilon_{2}$ such that $\varepsilon_{2} \leq 2$, we describe a drawing algorithm that computes $\left(0, \varepsilon_{2}\right)$-rectangle of influence drawings of binary trees in area $O\left(n^{2+f\left(\varepsilon_{2}\right)}\right)$, where $f\left(\varepsilon_{2}\right)$ is a logarithmic function that tends to infinity as $\varepsilon_{2}$ tends to zero, and $n$ is the number of vertices of the input tree. We recall that the study of the area required by proximity drawings of trees has received much attention in the graph drawing literature (see, e.g., [2]810]). Also, the design of area-efficient algorithms for approximate proximity drawings is one of the open problems stated by Evans et al. [9].

Open problems can be found in Section 5. In this section we also report some preliminary results that extend known techniques and that may be starting points for the proposed open problems. Some proofs are sketched or omitted for reasons of space.

\section{$2\left(\varepsilon_{1}, \varepsilon_{2}\right)$-Rectangle of Influence Drawings}

We say that a region of the plane is open if it is an open set, closed otherwise. Let $u=$ $(x(u), y(u))$ and $v=(x(v), y(v))$ be two points in the plane. Let $w=\left(\frac{x(u)+x(v)}{2}, \frac{y(u)+y(v)}{2}\right)$ and let $d_{x}=|x(u)-x(v)|$ and $d_{y}=|y(u)-y(v)|$. The open (closed) rectangle of influence of $u$ and $v$ is the open (closed) axis-aligned rectangle centered at $w$ with the horizontal side of length $d_{x}$ and the vertical side of length 


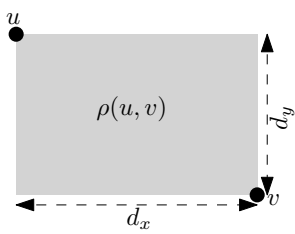

(a)

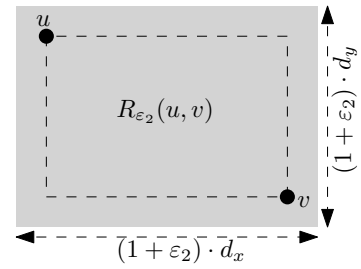

(b)

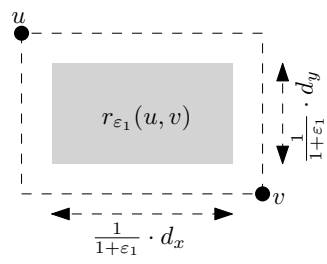

(c)

Fig. 1. (a) Rectangle of influence of $u$ and $v$; (b) $\varepsilon_{2}$-expanded rectangle of influence of $u$ and $v$; (c) $e_{2}$-shrunk rectangle of influence of $u$ and $v$.

$d_{y}$. The open (closed) $\varepsilon$-expanded rectangle of influence of $u$ and $v$ is the open (closed) axis-aligned rectangle centered at $w$ with the horizontal side of length $(1+\varepsilon) d_{x}$ and the vertical side of length $(1+\varepsilon) d_{y}$. The open (closed) $\varepsilon$-shrunk rectangle of influence of $u$ and $v$ is the axis-aligned open (closed) rectangle centered at $w$ with the horizontal side of length $\left(\frac{1}{1+\varepsilon}\right) d_{x}$ and the vertical side of length $\left(\frac{1}{1+\varepsilon}\right) d_{y}$.

The open (resp., closed) rectangle of influence of $u$ and $v$ is denoted as $\rho(u, v)$ (resp., $\rho[u, v]$ ). The open (resp., closed) expanded rectangle of influence of $u$ and $v$ is denoted as $R_{\varepsilon}(u, v)$ (resp., $R_{\varepsilon}[u, v]$ ). The open (resp., closed) shrunk rectangle of influence of $u$ and $v$ is denoted as $r_{\varepsilon}(u, v)$ (resp., $r_{\varepsilon}[u, v]$ ).

Let $G$ be a planar graph. A open (closed) $\left(\varepsilon_{1}, \varepsilon_{2}\right)$ rectangle of influence drawing $\Gamma$ of $G$ is a planar straight-line drawing of $G$ such that: (i) for every pair of vertices $u$ and $v$ that are adjacent in $G, r_{\varepsilon_{1}}(u, v)\left(r_{\varepsilon_{1}}[u, v]\right)$ does not contain any other vertex of $G$; (ii) for every pair of vertices $u$ and $v$ that are not adjacent in $G, R_{\varepsilon_{2}}(u, v)\left(R_{\varepsilon_{2}}[u, v]\right)$ contains at least one vertex of $G$. A rectangle of influence drawing will be called in the following a RID. Note that if two vertices are either horizontally or vertically aligned, then their rectangle of influence as well as its expanded and shrunk versions are degenerate rectangles. As a consequence, in the open model all such rectangles are empty sets. Following the assumption made by Liotta et al. [13] we assume that if $u, v$ and $w$ are points on the same horizontal (vertical) line and if $w$ lies between $u$ and $v$ then $\rho(u, v)$ contains $w$ and $R_{e}(u, v)$ contains $w$ for every $\varepsilon>0$, while $r_{\varepsilon}(u, v)$ contains $w$ if $w$ lies between the two endpoints of the segment representing $r_{\varepsilon}(u, v)$. In other words, dealing with degenerate rectangles, we treat the open ones like the closed ones.

By extending techniques of Evans et al. [9] to $\varepsilon_{1}$-shrunk rectangle of influence and $\varepsilon_{2}$-expanded rectangle of influence we can prove that every planar graph has both an open and closed $\left(\varepsilon_{1}, \varepsilon_{2}\right)$-RID for every $\varepsilon_{1}, \varepsilon_{2}>0$. On the other hand if one of the two parameters $\varepsilon_{1}$ and $\varepsilon_{2}$ is equal to 0 , then an $\left(\varepsilon_{1}, \varepsilon_{2}\right)$-RID may not exist.

Theorem 1. Every planar graph admits both an open and closed $\left(\varepsilon_{1}, \varepsilon_{2}\right)$-RID for every positive values of $\varepsilon_{1}, \varepsilon_{2}$. Also, there exist planar graphs that do not have a closed (open) $\left(\varepsilon_{1}, 0\right)-R I D$ and graphs that do not have a closed (open) $\left(0, \varepsilon_{2}\right)$-RID.

Sketch of Proof: In this sketch of proof we only describe how to compute a (closed and open) $\left(\varepsilon_{1}, \varepsilon_{2}\right)$-RID of a planar graph $G$, for every positive values of $\varepsilon_{1}, \varepsilon_{2}$. The drawing is constructed a vertex per time processing, vertices according to the canonical 
order. Augment $G$ to a maximal planar graph $G^{\prime}$ and compute a canonical ordering $v_{1}, v_{2}, \ldots, v_{n}$ of $G^{\prime}$ such that the edge $\left(v_{1}, v_{2}\right)$ is an edge of $G$. Let $G_{i}$ be the subgraph of $G^{\prime}$ induced by $V_{i}=\left\{v_{1}, v_{2}, \ldots, v_{i}\right\}$. We construct a drawing $\Gamma_{i}$ of $G_{i}$ by induction so that for every $i \geq 2$ : (a) $\Gamma_{i}$ is an embedding preserving open and closed $\left(\varepsilon_{1}, \varepsilon_{2}\right)$ RID of $G_{i}$; (b) all the vertices on the external face of $G_{i}$ are visible from the north-east direction, i.e., the straight line with slope one passing through each vertex $v_{j}$ on the external face of $\Gamma_{i}$ does not contain any vertex distinct from $v_{j}$ to the right of $v_{j}$. These properties can be satisfied for $i=2$ by placing $v_{1}$ at $(-1,1)$ and $v_{2}$ at $(1,-1)$. Assume we have a drawing $\Gamma_{i}$ of $G_{i}$ satisfying the above properties; we show how to compute a drawing $\Gamma_{i+1}$ of $G_{i+1}$ satisfying the same properties. Let $d$ be the diameter of the smallest disk $D$ enclosing $\Gamma_{i}$, and let $\sigma_{h}$ and $\sigma_{v}$ be a horizontal and a vertical line such that $D$ is contained in the third quadrant with respect to the point $p$ common to $\sigma_{h}$ and $\sigma_{v}$. We place vertex $v_{i+1}$ in the first quadrant with respect to $p$ far enough to guarantee that: (i) for every vertex $v_{j} \in V_{i}, r_{\varepsilon_{1}}\left[v_{i+1}, v_{j}\right]$ does not contain any vertex distinct from $v_{i+1}$ and $v_{j}$; (ii) for every vertex $v_{j} \in V_{i}, R_{\varepsilon_{2}}\left(v_{i+1}, v_{j}\right)$ contains at least one vertex distinct from $v_{i+1}$ and $v_{j}$; (iii) for every pair of vertices $v_{j}, v_{k} \in V_{i}$, we have $r_{\varepsilon_{1}}\left[v_{j}, v_{k}\right]$ does not contain $v_{i+1}$. Notice that properties (i) and (ii) imply that every vertex $v_{j} \in V_{i}$ can be both adjacent and non adjacent to $v_{i+1}$; thus we can make $v_{i+1}$ adjacent to the vertices of $V_{i}$ that are adjacent to $v_{i+1}$ in $G$, and not adjacent to the others. Let $u_{1}, u_{2}, \ldots, u_{k}$ be the vertices on the boundary of the external face of $G_{i}$ that are adjacent to $v_{i+1}$ in $G_{i+1}$ in their clockwise order along the boundary of the external face. Let $\sigma_{j}$ be the straight-line with slope one passing through $u_{j}(j=1,2, \ldots, k)$; we place $v_{i+1}$ within the strip bounded by $\sigma_{1}$ and $\sigma_{k}$ (this choice guarantees property (b)) so that its horizontal and vertical distance from $p$ is at least $\max \left\{\frac{2}{\varepsilon_{2}} d, \frac{2\left(1+\varepsilon_{1}\right)}{\varepsilon_{1}} d\right\}$. In addition we place $v_{i+1}$ far enough in order to guarantee planarity (note that there must exist a distance that guarantee planarity because $\Gamma_{i}$ satisfies property (b)). The proof that (i), (ii), and (iii) hold for $\Gamma_{i+1}$ is omitted.

Based on Theorem 1, it is worth investigating subclasses of the planar graphs that admit $\left(\varepsilon_{1}, \varepsilon_{2}\right)$-RIDs for either $\varepsilon_{1}=0$ or $\varepsilon_{2}=0$. In the following we describe different techniques to compute $\left(0, \varepsilon_{2}\right)$-RIDs of outerplanar graphs that are a classical subject of investigation in proximity drawability [12].

\section{$3\left(0, \varepsilon_{2}\right)$-RIDs of Outerplanar Graphs: A General Technique}

In this section we prove that every outerplanar graph admits both an open and a closed $\left(0, \varepsilon_{2}\right)$-RID for every $\varepsilon_{2}>0$. Our technique draws an outerplanar graph $G$ by first computing a drawing of a BFS tree of $G$ and by then adding the remaining edges. For this reason we first prove that every tree admits both an open and a closed $\left(0, \varepsilon_{2}\right)$-RID for every $\varepsilon_{2}>0$.

Lemma 1. Let $T$ be a star. For every $\varepsilon_{2}>0, T$ admits both a closed and an open $\left(0, \varepsilon_{2}\right)-R I D \Gamma$ such that: (i) no leaf of $T$ is on the bottom or left side of the bounding box $\mathcal{B}$ of $\Gamma$; (ii) the internal vertex of $T$ is on the bottom-left corner of $\mathcal{B}$.

Proof. Let $u$ be the unique internal vertex of $T$ and let $u_{1}, u_{2}, \ldots, u_{k}$ be the leaves of $T$ in the clockwise order around $u$. Let $p$ be a real number such that $p>\frac{2}{\varepsilon_{2}}+1$. Place 
$u$ at $(0,0)$ and each vertex $u_{i}(1 \leq i \leq k)$ at $\left(p^{i-1}, p^{k-i}\right)$. Properties $(i)$ and $(i i)$ hold by construction. We show that the drawing is both an open and a closed $\left(0, \varepsilon_{2}\right)$-RID.

Consider an edge $\left(u, u_{i}\right)$ of $T$. We prove that $\rho\left[u, u_{i}\right]$ does not contain any vertex distinct from $u$ and $u_{i}$. This implies that also $\rho\left(u, u_{i}\right)$ does not contain any vertex distinct from $u$ and $u_{i}$. A point $q$ belongs to $\rho\left[u, u_{i}\right]$ if its $x$-coordinate $x(q)$ is such that $0 \leq x(q) \leq p^{i-1}$ and its $y$-coordinate $y(q)$ is such that $0 \leq y(q) \leq p^{k-i}$. Each vertex $u_{j}$ with $j<i$ has $y\left(u_{j}\right)=p^{k-j}>p^{k-i}$ and therefore $u_{j} \notin \rho\left[u, u_{i}\right]$. Each vertex $u_{j}$ with $j>i$ has $x\left(u_{j}\right)=p^{j-1}>p^{i-1}$ and therefore $u_{j} \notin \rho\left[u, u_{i}\right]$.

Let $u_{i}$ and $u_{j}$ with $j>i$ be two vertices that are not adjacent in $T$. We prove that $R_{\varepsilon_{2}}\left(u_{i}, u_{j}\right)$ contains at least one vertex distinct from $u_{i}$ and $u_{j}$. This implies that also $R_{\varepsilon_{2}}\left[u_{i}, u_{j}\right]$ contains at least one vertex distinct from $u_{i}$ and $u_{j}$. Let $q$ be the bottom-left corner of $R_{\varepsilon_{2}}\left(u_{i}, u_{i+1}\right)$; we have $x(q)=x\left(u_{i}\right)-\Delta_{x}$, where $\Delta_{x}=\frac{\varepsilon_{2}}{2}\left(x\left(u_{j}\right)-x\left(u_{i}\right)\right)$, which can be rewritten as $x(q)=p^{i-1}-\frac{\varepsilon_{2}}{2}\left(p^{j-1}-p^{i-1}\right)$. Since $j \geq i+1$, we have $x(q) \leq p^{i-1}-\frac{\varepsilon_{2}}{2}\left(p^{i}-p^{i-1}\right)=p^{i-1}\left(1-\frac{\varepsilon_{2}}{2}(p-1)\right)$. From $p>\frac{2}{\varepsilon_{2}}+1$ it follows $x(q)<0$. Analogously, we have $y(q)=y\left(u_{j}\right)-\Delta_{y}$ with $\Delta_{y}=\frac{\varepsilon_{2}}{2}\left(y\left(u_{i}\right)-y\left(u_{j}\right)\right)$, which gives $y(q)=p^{k-j}-\frac{\varepsilon_{2}}{2}\left(p^{k-i}-p^{k-j}\right)$. Since $j \geq i+1$, we have $y(q) \leq$ $p^{k-j}-\frac{\varepsilon_{2}}{2}\left(p^{k-j+1}-p^{k-j}\right)=p^{k-j}\left(1-\frac{\varepsilon_{2}}{2}(p-1)\right)$. So, also in this case $y(q)<0$. It follows that $R_{\varepsilon_{2}}\left(u_{i}, u_{j}\right)$ contains the point $(0,0)$ and therefore $u$.

Lemma 2. Every tree admits both an open and a closed $\left(0, \varepsilon_{2}\right)$-RID for every $\varepsilon_{2}>0$.

Sketch of Proof: We prove by induction that $T$ admits both a (closed and open) $\left(0, \varepsilon_{2}\right)$ RID $\Gamma$ with the following additional properties: $(i)$ no vertex except for the root is drawn on the bottom or left side of the bounding box of $\Gamma$; $(i i)$ the root of $T$ is drawn at the bottom-left corner of the bounding box of $\Gamma$. The induction is on the number of internal vertices $n_{i}$ of $T$.

If $n_{i}=1$, then $T$ is a star and therefore it admits a (closed or open) $\left(0, \varepsilon_{2}\right)$-RID with properties $(i)$ and $(i i)$ by Lemma 1. If $n_{i}>1$, let $u$ be the root of $T$ and let $u_{1}, u_{2}, \ldots, u_{k}$ be the children of $u$. The subtree $T_{u}$ induced by $u$ and its children is a star and can be drawn according to Lemma 1 Let $\gamma_{u}$ be the drawing of $T_{u}$ and let $T_{u_{i}}$ ( $i=1,2, \ldots, k)$ be the subtree rooted at $u_{i}$. By induction, every $T_{i}$ admits a (closed and open) $\left(0, \varepsilon_{2}\right)$-RID $\Gamma_{i}$ with properties $(i)$ and $(i i)$. Each $\Gamma_{i}$ is scaled down so that its bounding box has width $W_{i}<\frac{\varepsilon_{2}}{2+\varepsilon_{2}} p^{i-1}(p-1)$ and height $H_{i}<\frac{\varepsilon_{2}}{2+\varepsilon_{2}} p^{k-i}(p-1)$ and it is placed so that it bottom-left corner coincides with the point representing $u_{i}$ in $\Gamma_{u}$ (by property $(i i) u_{i}$ is represented by a unique point in the resulting drawing). The proof of the correctness of this drawing technique is omitted. Properties $(i)$ and $(i i)$ hold by construction.

Lemma 3. Every connected outerplanar graph admits both an open and a closed $\left(0, \varepsilon_{2}\right)-R I D$ for every $\varepsilon_{2}>0$.

Sketch of Proof: Let $G$ be an outerplanar graph. Consider an outerplanar embedding of $G$ and perform a BFS traversal of $G$ such that the neighbors of each vertex are scanned according to the counterclockwise order defined by the embedding of $G$. Let $T$ be the resulting BFS tree. We compute a (closed and open) $\left(0, \varepsilon_{2}\right)$-RID of $T$ by using the technique described in the proof of Lemma 2. Denote by $u_{i, 1}, u_{i, 2}, \ldots, u_{i, n_{i}}$ the vertices at depth $i$ in $T$ ordered according to their left-to-right order (corresponding to 
counterclockwise order around each vertex defined by the planar embedding of $G$ ). Let $(v, w)$ be an edge of $G$ that is not in $T$. It is easy to see that two cases are possible: Case 1. $v=u_{i, j}$ and $w=u_{i, j+1}\left(i \geq 0,0 \leq j \leq n_{i}\right)$; Case 2. $v=u_{i, j}$ and $w=u_{i-1, k}$, where $u_{i-1, k-1}$ is the parent of $u_{i, j}\left(i \geq 1,1 \leq k \leq n_{i-1}, 0 \leq j \leq n_{i}\right)$. In this case $u_{i, j}$ is the rightmost child of $u_{i-1, k-1}$. It is possible to show that in both cases the edge $(v, w)$ can be added to the drawing.

Lemma 3 states that an outerplanar graph admits a (closed and open) $\left(0, \varepsilon_{2}\right)$-RID if it is connected; for a non-connected outerplanar graph $G$, it is possible to prove that if all the connected components of $G$ admit a (closed and open) $\left(0, \varepsilon_{2}\right)$-RID and $G$ has at least one edge, then $G$ admits a (closed and open) $\left(0, \varepsilon_{2}\right)$-RID. The following theorem holds.

Theorem 2. Every outerplanar graph with at least one edge admits both a closed and an open $\left(0, \varepsilon_{2}\right)$-RID for every $\varepsilon_{2}>0$.

We conclude the section by recalling that there exist outerplanar graphs that do not have a $(0,0)$-RID both in the open and closed model, while all outerplanar graphs have a $(0, \infty)$-RID [513]. Hence, Theorem2 extends the $\left(0, \varepsilon_{2}\right)$-rectangle of influence drawability of outerplanar graphs to all positive values of $\varepsilon_{2}$.

\section{$4\left(0, \varepsilon_{2}\right)$-RIDs of Outerplanar Graphs: Polynomial Area Constructions}

The drawing construction in the proof of Theorem 2 produces drawings whose area is exponential in the number of vertices of the input graph. In this section we give two different polynomial area drawing techniques for $\left(0, \varepsilon_{2}\right)$-RIDs of outerplanar graphs. The first one applies to every outerplanar graph (it is indeed even more general) but it works only for $\varepsilon_{2}>2$; the second one assumes that $0<\varepsilon_{2} \leq 2$ but it can be applied only to trees with maximum vertex degree three.

A $k$-track graph $G=(V, E, \phi)$ is an undirected graph with a level assignment $\phi$ : $V \rightarrow\{1,2, \ldots, k\}(1 \leq k \leq|V|)$ [3]. Each set $V_{i}=\{v \in V \mid \phi(v)=i\}$ is called a level. A $k$-track graph is proper if $|\phi(u)-\phi(v)| \leq 1$ for every edge $(u, v) \in E$. A $k$ track graph $G$ is $k$-track planar (or simply track planar) if there exist a planar drawing $\Gamma$ such that the vertices of level $V_{i}$ are drawn on the horizontal line $y=i$. The set of the linear orderings of the vertices of each level is the $k$-track planar embedding (or simply track-planar embedding) of $\Gamma$. Let $\sigma_{1}, \sigma_{2}, \ldots, \sigma_{k}$ be a set of parallel lines. It is easy to see that, given a $k$-track-planar embedding $\gamma$ of a $k$-track-planar graph $G$, any drawing of $G$ such that the vertices level $V_{i}$ are drawn on $\sigma_{i}(1 \leq i \leq k)$ according to the linear ordering defined by $\gamma$ is planar. Also, if $u$ and $v$ are vertices of the same level and they are adjacent then in any track-planar embedding $u$ and $v$ are consecutive. It is easy to see that an outerplanar graph is a $k$-track graph. Namely, if we perform a BFS traversal of an outerplanar graph $G$ as described in the proof of Lemma 3, we obtain a track planar embedding of $G$ whose levels corresponds to the levels of the BFS tree.

Theorem 3. Every proper track planar graph with $n$ vertices admits both an open and a closed $\left(0, \varepsilon_{2}\right)-R I D$ in $O\left(n^{4}\right)$ area for every $\varepsilon_{2}>2$. 
Sketch of Proof: Let $G$ be a proper $k$-track planar graph and let $\gamma$ be a $k$-track planar embedding of $G$. Assume $G$ is connected and there is no level with exactly two vertices. Denote by $u_{i, 1}, u_{i, 2}, \ldots, u_{i, n_{i}}$ the vertices of level $i(1 \leq i \leq k)$ according to the linear ordering defined by $\gamma$. Draw vertex $u_{i, j}$ at point $\left(N_{i-1}+j, N_{i}-j+1\right)$, where $N_{i}=\sum_{h=1}^{i} n_{h}$ if $i>0$ and $N_{0}=0$. It is easy to check that the resulting drawing is both an open and a closed $\left(0, \varepsilon_{2}\right)$-RID for every $\varepsilon_{2}>2$ and that the area is $O\left(n^{4}\right)$. The proof when $G$ is not connected and/or it has a level with exactly two vertices follows a similar approach.

We describe now another drawing technique that computes $\left(0, \varepsilon_{2}\right)$-RIDs of trees with maximum vertex degree three in area that is polynomial for every given $\varepsilon_{2}$. We decompose the tree into subtrees of smaller size by means of a greedy path decomposition. Let $T$ be a rooted tree such that each vertex has at most $d$ children $(d \geq 2)$, and let $u_{1}$ be the root of $T$. A greedy path of $T$ is a path $u_{1}, u_{2}, \ldots, u_{h}$ connecting the root $u_{1}$ to a leaf $u_{h}$ and such that $u_{i}$ is the root of the largest subtree rooted at $u_{i-1}(2 \leq i \leq h)$. A greedy path decomposition of a rooted tree $T$ consists of recursively identifying greedy paths and on removing them so to decompose the tree into rooted subtrees of smaller size. The decomposition ends when the tree is a path (possibly consisting of a single vertex). Greedy paths decompositions of rooted trees have been used in many papers to compute compact drawings of binary trees (see, e.g., [8[10]).

Let $T$ be a tree with a given greedy path decomposition and let $T^{\prime}$ be a subtree of $T$. The greedy depth of $T^{\prime}$ (with respect to the given decomposition) is denoted as $\gamma\left(T^{\prime}\right)$, and defined as follows: (i) If $T^{\prime}$ is a path, $\gamma\left(T^{\prime}\right)=1$; $(i i)$ otherwise, $\gamma\left(T^{\prime}\right)=$ $\max _{i}\left\{\gamma\left(T_{i}\right)\right\}+1$, where each $T_{i}$ is a tree obtained from $T^{\prime}$ by removing its greedy path for the given decomposition. Intuitively, the greedy depth of a tree for a given greedy path decomposition is the depth of the recursion in the decomposition process.

Theorem 4. Every tree with $n$ vertices and vertex degree at most three admits both an open and a closed $\left(0, \varepsilon_{2}\right)-R I D$ in $O\left(n^{2+3 \log _{2}\left(1+\frac{8}{\varepsilon_{2}}\right)}\right)$ area for every $0<\varepsilon_{2} \leq 2$.

Sketch of Proof: We describe a drawing algorithm assuming that $T$ is rooted and each internal vertex of $T$ has at most two children. This assumption is not restrictive. Indeed, if $T$ is a tree of degree at most three, we can always root $T$ at a leaf. In this way every internal vertex of $T$ has at most two children.

Let $\varepsilon=\frac{\varepsilon_{2}}{2}$, let $c_{\varepsilon}=\left(1+\frac{4}{\varepsilon}\right)$ and let $\mathcal{W}(n)$ and $\mathcal{H}(n)$ be defined as follows:

$$
\mathcal{W}(n)=\left\{\begin{array}{ll}
2 & \text { if } n=0 \\
4 n c_{\varepsilon} \log _{2} n & \text { if } n>0
\end{array} \quad \mathcal{H}(n)=n c_{\varepsilon}^{\log _{2} n}\left(1+4 \frac{c_{\varepsilon}^{\log _{2} n}-1}{c_{\varepsilon}-1}\right)\right.
$$

The drawing algorithm applies a recursive construction based on a greedy path decomposition. Let $T^{\prime}=\left(V^{\prime}, E^{\prime}\right)$ be a subtree of $T$ such that $\gamma\left(T^{\prime}\right)=i\left(1 \leq i \leq\left\lceil\log _{2} n\right\rceil\right)$. Let $\Pi$ be the greedy path of $T^{\prime}$. The algorithm constructs a drawing $\Gamma^{\prime}$ of $T^{\prime}$ by composing the drawings of all trees obtained from $T^{\prime}$ by removing $\Pi$. Let $n^{\prime}$ be the number of vertices of $T^{\prime}$. The algorithm maintains the following invariants for $\Gamma^{\prime}$ : (I1) $\Gamma^{\prime}$ is both an open and closed $\left(0, \varepsilon_{2}\right)$-RID of $T^{\prime}$; (I2) the root $v^{\prime}$ of $T^{\prime}$ is drawn on the left 
border of $\Gamma^{\prime}$ and no vertex is drawn on the same border below $v^{\prime}$; (I3) $\Gamma^{\prime}$ is completely contained in a bounding box of size $W\left(\Gamma^{\prime}\right) \leq \mathcal{W}\left(n^{\prime}\right)$ and height $H\left(\Gamma^{\prime}\right) \leq \mathcal{H}\left(n^{\prime}\right)$.

We now prove that $\Gamma^{\prime}$ can be constructed. The proof is by induction on the greedy depth $i$ of $T^{\prime}$. Denote by $u_{1}, u_{2}, \ldots, u_{h}$ the vertices of the greedy path $\Pi$ of $T^{\prime}$, and let $v_{j}$ be the child of $u_{j}$ that is not in $\Pi(1 \leq j \leq h)$. We denote as $n_{j}$ the number of vertices in the subtree rooted at $v_{j}$. If vertex $v_{j}$ (and the corresponding subtree) does not exist for some $j$, we set $n_{j}=0$. In particular, if the greedy depth of $T^{\prime} i$ is one (i.e., we are in the base case of the induction) $n_{j}=0$ for every $j$. The vertices $u_{1}, u_{2}, \ldots, u_{h}$ are drawn along a straight line $r$ with slope -1 , in this order from left to right. The horizontal and vertical distance between $u_{j}$ and $u_{j+1}(1 \leq j \leq h-1)$ is denoted by $\ell_{j}$ and it is equal to $\mathcal{W}\left(n_{j}\right)+\frac{2}{\varepsilon} \max \left\{\mathcal{W}\left(n_{j}\right), \mathcal{W}\left(n_{j+1}\right)\right\}$. If $i=1$, i.e., we are in the base case of the induction, $T^{\prime}$ coincides with the greedy path $\Pi$ and thus $T^{\prime}$ is completely drawn. Notice that, in this case, the vertices of $T^{\prime}$ are equispaced along $r$ and the horizontal and vertical distance between two consecutive vertices is $\ell_{j}=\mathcal{W}\left(n_{j}\right)+\frac{2}{\varepsilon} \max \left\{\mathcal{W}\left(n_{j}\right), \mathcal{W}\left(n_{j+1}\right)\right\}=2\left(1+\frac{2}{\varepsilon}\right)$ because $\mathcal{W}(0)=2$.

If $i>1$, i.e., we are in the inductive case, assume that each subtree with greedy depth $i-1(i>1)$ admits a drawing satisfying Invariants (I1), (I2), and (I3). We complete the construction of $\Gamma^{\prime}$ as follows (see also Figure 2). For every $1 \leq j \leq h-1$, if $n_{j}>0$, denote by $\Gamma_{j}$ the drawing of the subtree rooted at $v_{j}(1 \leq j \leq h-1)$. Each drawing $\Gamma_{j}$ is placed above the line $r$. More precisely, if $n_{j}=1$, then $\Gamma_{j}$ is placed one unit above and one unit to the left of $u_{j}$; if $n_{j}>1$, then $\Gamma_{j}$ is placed in such a way that $v_{j}$ is vertically aligned with $u_{j}$ and above it; the length of the edge $\left(u_{j}, v_{j}\right)$ is denoted as $d_{j}$ and is defined as follows. Let $j^{-}$be the largest index such that $j^{-}<j$ and $n_{j^{-}}>1$. Notice that $j^{-}$may not exist for some values of $j$. If $j^{-}$is defined, then he value of $d_{j}$ is chosen in such a way that the bottommost vertex of $\Gamma_{j}$ is above the topmost vertex of $\Gamma_{j^{-}}$at a vertical distance of $\frac{2}{\varepsilon} \max \left\{\mathcal{H}\left(n_{j}\right), \mathcal{H}\left(n_{j^{-}}\right)\right\}$; if $j^{-}$is not defined, then $d_{j}$ is chosen in such a way that the bottommost vertex of $\Gamma_{j}$ is above the horizontal line $y=y\left(u_{1}\right)$ at a distance of $\frac{2}{\varepsilon} \mathcal{H}\left(n_{j}\right)$.

The proof that $\Gamma^{\prime}$ is both an open and a closed $\left(0, \varepsilon_{2}\right)$-RID of $T^{\prime}$, i.e., that Invariant (I1) holds are omitted. Invariant (I2) holds by construction because nothing is drawn below the line $r$ that contains the greedy path. We now prove that Invariant (I3) holds. If we are in the base case of the induction, then $T^{\prime}$ coincides with the greedy path and therefore both the width and the height of $\Gamma^{\prime}$ are equal to the width of the greedy path which is $\sum_{j=1}^{n-1} \ell_{j}=\sum_{j=1}^{n-1} 2\left(1+\frac{2}{\varepsilon}\right)=2(n-1)\left(1+\frac{2}{\varepsilon}\right)$. We have $2(n-1)\left(1+\frac{2}{\varepsilon}\right) \leq 4 n\left(1+\frac{4}{\varepsilon}\right)^{\log _{2} n}=4 n c_{\varepsilon}^{\log _{2} n}=\mathcal{W}(n)$ for every $n>0$; also, $2(n-1)\left(1+\frac{2}{\varepsilon}\right) \leq n c_{\varepsilon}^{\log _{2} n}\left(1+4 \frac{c_{\varepsilon}^{\log _{2} n}-1}{c_{\varepsilon}-1}\right)=\mathcal{H}(n)$ for every $n>0$. Thus Invariant (I3) holds in the base case of the induction.

Consider now the inductive case. The width $W\left(\Gamma^{\prime}\right)$ of $\Gamma^{\prime}$ is equal to the width of the greedy path which is $\sum_{j=1}^{h-1} \ell_{j}=\sum_{j=1}^{h-1}\left(\mathcal{W}\left(n_{j}\right)+\frac{2}{\varepsilon} \max \left\{\mathcal{W}\left(n_{j}\right), \mathcal{W}\left(n_{j+1}\right)\right\}\right) \leq$ $\sum_{j=1}^{h-1} \mathcal{W}\left(n_{j}\right)+\frac{2}{\varepsilon} \sum_{j=1}^{h-1} \mathcal{W}\left(n_{j}\right)+\frac{2}{\varepsilon} \sum_{j=1}^{h-1} \mathcal{W}\left(n_{j+1}\right) \leq \sum_{j=1}^{h} \mathcal{W}\left(n_{j}\right)+$ $\frac{4}{\varepsilon} \sum_{j=1}^{h} \mathcal{W}\left(n_{j}\right)=\left(1+\frac{4}{\varepsilon}\right) \sum_{j=1}^{h} \mathcal{W}\left(n_{j}\right)=c_{\varepsilon} \sum_{j=1}^{h} \mathcal{W}\left(n_{j}\right)$.

Let $J_{1}=\left\{j \mid 1 \leq j \leq h \wedge n_{j}>0\right\}$ and let $J_{2}=\left\{j \mid 1 \leq j \leq h \wedge n_{j}=\right.$ $0\}$; we have: $W\left(\Gamma^{\prime}\right) \leq c_{\varepsilon} \sum_{j=1}^{h} \mathcal{W}\left(n_{j}\right)=c_{\varepsilon}\left(\sum_{j \in J_{1}} \mathcal{W}\left(n_{j}\right)+\sum_{j \in J_{2}} \mathcal{W}\left(n_{j}\right)\right)=$ 


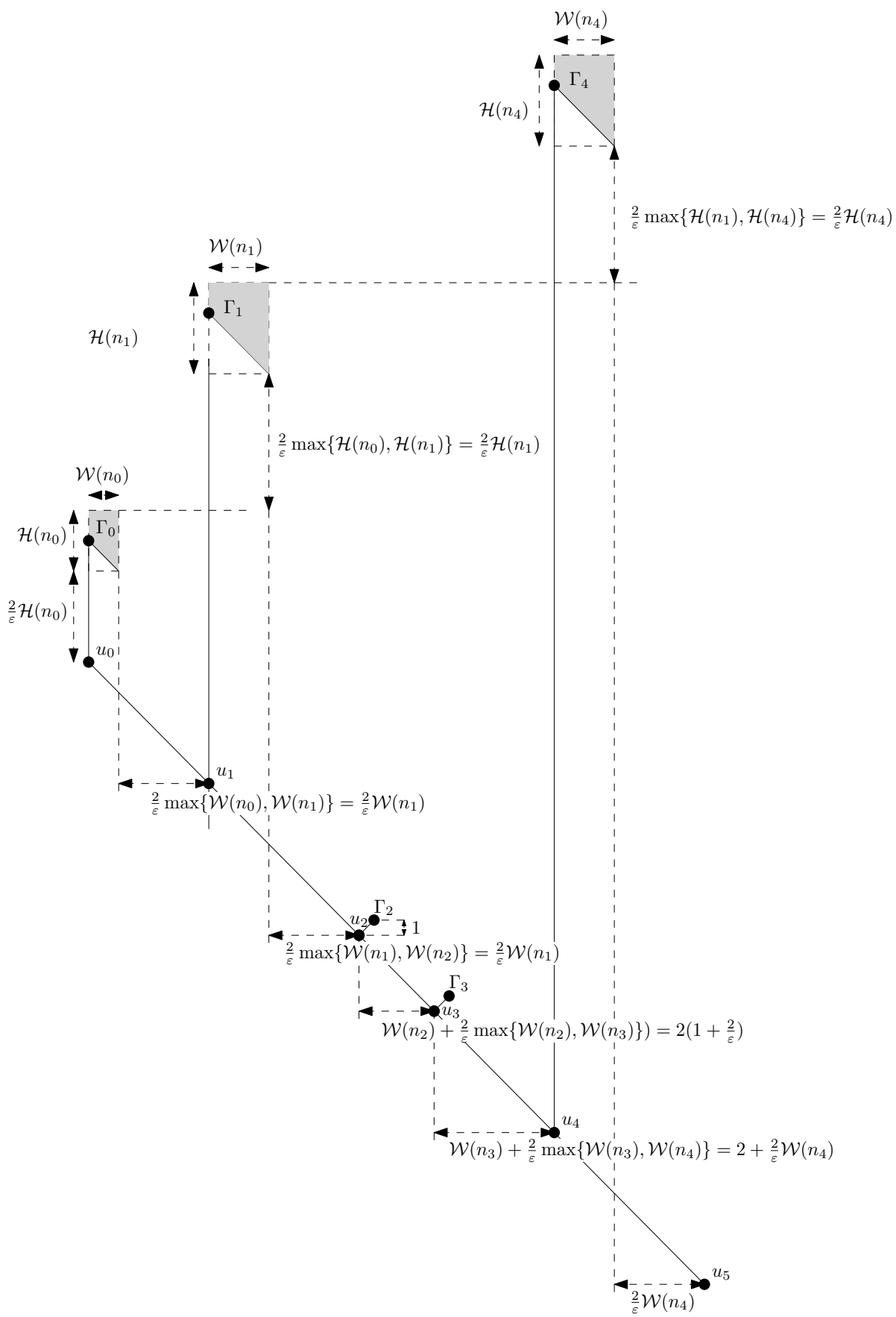

Fig. 2. Illustration of the drawing technique to compute a (closed or open) $\left(0, \varepsilon_{2}\right)$-RID of a tree with maximum vertex degree three 
$c_{\varepsilon}\left(\sum_{j \in J_{1}} 4 n_{j} c_{\varepsilon}^{\log _{2} n_{j}}+\sum_{j \in J_{2}} 2\right)$. According to the definition of greedy path $n_{j} \leq$

$\frac{n}{2}$. Also, $\sum_{j \in J_{2}} 2 \leq \sum_{j \in J_{2}} 4 c_{\varepsilon}^{\log _{2} \frac{n}{2}}$ because $c_{\varepsilon}>1$ and $n>1$. Thus, $W\left(\Gamma^{\prime}\right) \leq$ $4 c_{\varepsilon}^{\log _{2} n}\left(\sum_{j \in J_{1}} n_{i}+\sum_{j \in J_{2}} 1\right) \leq 4 c_{\varepsilon}^{\log _{2} n}(n-h+h)=4 n c_{\varepsilon}^{\log _{2} n}=\mathcal{W}(n)$.

The height $H\left(\Gamma^{\prime}\right)$ is equal to the width of $\Gamma^{\prime}$ plus an extra amount given by the height occupied by the subtrees of size larger than one which are above the horizontal line $y=y\left(u_{1}\right)$. Thus,

$H\left(\Gamma^{\prime}\right) \leq W\left(\Gamma^{\prime}\right)+\sum_{j \in J_{1}} \mathcal{H}\left(n_{j}\right)+\frac{2}{\varepsilon} \sum_{j \in J_{1}} \max \left\{\mathcal{H}\left(n_{j}\right), \mathcal{H}\left(n_{j^{-}}\right)\right\} \leq W\left(\Gamma^{\prime}\right)+$ $\sum_{j \in J_{1}} \mathcal{H}\left(n_{j}\right)+\frac{4}{\varepsilon} \sum_{j \in J_{1}} \mathcal{H}\left(n_{j}\right)=W\left(\Gamma^{\prime}\right)+c_{\varepsilon} \sum_{j \in J_{1}} \mathcal{H}\left(n_{j}\right) \leq$

$4 n c_{\varepsilon}^{\log _{2} n}+c_{\varepsilon} \sum_{j \in J_{1}} n_{j} c_{\varepsilon}^{\log _{2} n_{j}}\left(1+4 \frac{c_{\varepsilon}^{\log _{2} n_{j}}-1}{c_{\varepsilon}-1}\right)=4 n c_{\varepsilon}^{\log _{2} n}+c_{\varepsilon} \sum_{j \in J_{1}} n_{j} c_{\varepsilon}^{\log _{2} n_{j}}+$ $c_{\varepsilon} \sum_{j \in J_{1}} 4 n_{j} c_{\varepsilon}^{\log _{2} n_{j}} \frac{c_{\varepsilon} \log _{2} n_{j}-1}{c_{\varepsilon}-1}$. Again, $n_{j} \leq \frac{n}{2}$ and $\sum_{j \in J_{1}} n_{j} \leq n$. Therefore $H\left(\Gamma^{\prime}\right) \leq$ $4 n c_{\varepsilon}^{\log _{2} n}+n c_{\varepsilon}^{\log _{2} n}+4 n c_{\varepsilon}^{\log _{2} n} \frac{c_{\varepsilon}^{\log _{2} \frac{n}{2}}-1}{c_{\varepsilon}-1}=n c_{\varepsilon}^{\log _{2} n}\left(5+4 \frac{c_{\varepsilon}^{\log _{2} \frac{n}{2}}-1}{c_{\varepsilon}-1}\right)$. By simple calculations it can be proved that $5+4 \frac{c_{\varepsilon}^{\log _{2} \frac{n}{2}}-1}{c_{\varepsilon}-1} \leq 1+4 \frac{c_{\varepsilon}^{\log _{2} n}-1}{c_{\varepsilon}-1}$ for $n \geq 2$, which is always true in the inductive case. Hence $H\left(\Gamma^{\prime}\right) \leq n c_{\varepsilon}^{\log _{2} n}\left(1+4 \frac{c_{\varepsilon}^{\log _{2} n}-1}{c_{\varepsilon}-1}\right)=\mathcal{H}(n)$ and Invariant ( I3) holds also in the inductive case.

In order to prove the bound on the area of the drawing we observe that $\mathcal{W}(n)=$ $4 n c_{\varepsilon}^{\log _{2} n}=4 n^{1+\log _{2} c_{\varepsilon}}=O\left(n^{1+\log _{2} c_{\varepsilon}}\right)$ and, recalling that $c_{\varepsilon}>1, \mathcal{H}(n) \leq n c_{\varepsilon}^{\log _{2} n}(1+$ $\left.4 c_{\varepsilon}^{\log _{2} n}\right)=n^{1+\log _{2} c_{\varepsilon}}\left(1+4 n^{\log _{2} c_{\varepsilon}}\right)=O\left(n^{1+2 \log _{2} c_{\varepsilon}}\right)$. The area is at most $\mathcal{W}(n) \times$ $\mathcal{H}(n)=O\left(n^{2+3 \log _{2}\left(1+\frac{4}{\varepsilon}\right)}\right)=O\left(n^{2+3 \log _{2}\left(1+\frac{8}{\varepsilon_{2}}\right)}\right)$.

\section{Open Problems}

It would be interesting to study other families of planar graphs that admit a $\left(0, \varepsilon_{2}\right)$-RID for $\varepsilon_{2}>0$. For example, Biedl et al. prove that all graphs with no filled 3-cycles (NF3 graphs) have an open/closed $(0, \infty)$-RID [5]. Since outerplanar graphs have no filled 3 -cycles, Theorem 2 partially extends the result by Biedl et al. to all positive values of $\varepsilon_{2}$. Do all NF3 graphs admit a $\left(0, \varepsilon_{2}\right)$-rectangle of influence drawing for all values of $\varepsilon_{2}$ such that $\varepsilon_{2}>0$ ? A preliminary result in this direction is the following theorem, whose proof is an adaptation of the technique in [5].

Theorem 5. Every 4-connected internally triangulated plane graph whose outerface has at least 4 vertices admits both a closed and a open $\left(0, \varepsilon_{2}\right)$-RID for every $\varepsilon_{2}>0$.

Since $\left(\varepsilon_{1}, \varepsilon_{2}\right)$-RIDs exist for every planar graph if $\varepsilon_{1}>0$ and $\varepsilon_{2}>0$ (see Section 2), we focused on the case when $\varepsilon_{1}$ is zero. It is natural to investigate the problem for $\varepsilon_{2}=0$ and $\varepsilon_{1}>0$. A preliminary result in this direction is as follows.

Theorem 6. Every biconnected outerplanar graph admits both a closed and an open $\left(\varepsilon_{1}, 0\right)$-RID for every $\varepsilon_{1}>0$.

The area required by the drawing techniques of Theorem 5 and 6 is exponential. Thus it is worth studying polynomial area drawing constructions. 
Finally, for any given value $\varepsilon_{2}<2$, Theorem 4 shows how to compute a polynomial area drawing of a binary tree. It would be interesting to extend the result of Theorem 4 to larger families of outerplanar graphs.

\section{References}

1. Alamdari, S., Biedl, T.: Planar Open Rectangle-of-Influence Drawings with Non-aligned Frames. In: van Kreveld, M., Speckmann, B. (eds.) GD 2011. LNCS, vol. 7034, pp. 14-25. Springer, Heidelberg (2012)

2. Angelini, P., Bruckdorfer, T., Chiesa, M., Frati, F., Kaufmann, M., Squarcella, C.: On the Area Requirements of Euclidean Minimum Spanning Trees. In: Dehne, F., Iacono, J., Sack, J.-R. (eds.) WADS 2011. LNCS, vol. 6844, pp. 25-36. Springer, Heidelberg (2011)

3. Bachmaier, C., Brandenburg, F.J., Forster, M.: Track planarity testing and embedding. In: Proc. of SOFSEM 2004, vol. 2, pp. 3-17. MatFyzPress (2004)

4. de Berg, M.T., Carlsson, S., Overmars, M.H.: A general approach to dominance in the plane. J. Algorithms 13, 274-296 (1992)

5. Biedl, T., Bretscher, A., Meijer, H.: Rectangle of Influence Drawings of Graphs without Filled 3-Cycles. In: Kratochvíl, J. (ed.) GD 1999. LNCS, vol. 1731, pp. 359-368. Springer, Heidelberg (1999)

6. Bose, P., Lenhart, W., Liotta, G.: Characterizing proximity trees. Algorithmica 16, 83-110 (1996)

7. Di Battista, G., Liotta, G., Whitesides, S.: The strength of weak proximity. J. Discrete Algorithms 4(3), 384-400 (2006)

8. Di Giacomo, E., Didimo, W., Liotta, G., Meijer, H.: Drawing a tree as a minimum spanning tree approximation. J. Comput. Syst. Sci. 78(2), 491-503 (2012)

9. Evans, W., Gansner, E.R., Kaufmann, M., Liotta, G., Meijer, H., Spillner, A.: Approximate Proximity Drawings. In: van Kreveld, M., Speckmann, B. (eds.) GD 2011. LNCS, vol. 7034, pp. 166-178. Springer, Heidelberg (2011)

10. Frati, F., Kaufmann, M.: Polynomial area bounds for MST embeddings of trees. Comput. Geom. Theory and Applications 44(9), 529-543 (2011)

11. Ichino, M., Sklansky, J.: The relative neighborhood graph for mixed feature variables. Pattern Recognition 18(2), 161-167 (1985)

12. Liotta, G.: Proximity drawings. In: Tamassia, R. (ed.) Handbook of Graph Drawing and Visualization. CRC Press (to appear)

13. Liotta, G., Lubiw, A., Meijer, H., Whitesides, S.: The rectangle of influence drawability problem. Comput. Geom. Theory and Applications 10(1), 1-22 (1998)

14. Miura, K., Matsuno, T., Nishizeki, T.: Open rectangle-of-influence drawings of inner triangulated plane graphs. Discrete \& Computational Geometry 41(4), 643-670 (2009)

15. Miura, K., Nishizeki, T.: Rectangle-of-influence drawings of four-connected plane graphs. In: APVIS 2005. CRPIT, vol. 45, pp. 75-80. Australian Computer Society (2005)

16. Miura, K., Nakano, S.-I., Nishizeki, T.: Convex Grid Drawings of Four-Connected Plane Graphs. In: Lee, D.T., Teng, S.-H. (eds.) ISAAC 2000. LNCS, vol. 1969, pp. 254-265. Springer, Heidelberg (2000)

17. Overmars, M.H., Wood, D.: On rectangular visibility. J. Algorithms 9, 372-390 (1988)

18. Sadasivam, S., Zhang, H.: Closed rectangle-of-influence drawings for irreducible triangulations. Comput. Geom. Theory and Applications 44(1), 9-19 (2011)

19. Zhang, H., Vaidya, M.: On open rectangle-of-influence and rectangular dual drawings of plane graphs. Discrete Mathematics, Algorithms and Applications 1(3), 319-333 (2009) 